\title{
遷移沸騰熱伝達問題
}

\section{第三部 遷移沸騰熱伝達}

\author{
Transition Boiling Heat Transfer Part III \\ V. V. Kostiyuk, N. N. Borisov and I. Berlin \\ Moscow Aviation Institute, Moscow USSR
}

(Received July 24, 1978)

玉 田 紀 治* ・富山朔太 郎* 訳

\section{Synopsis :}

At present, method of transient cooldown is widely used for studying boiling process. This method was successfully applied to studies of film boiling of cryogenic liquids.

The aim of the present work is to determine temperature of heat exchange surface while investigating heat transfer by the method of transient cooldown. For this purpose it is proposed to approximate temperature curve cooldown with the help of continued smooth function.

\section{二次元座標系で表わした非定常逆熱伝達問題}

今日, 非定常逆熱伝達問題の解法は良く知られてい る。これら解法のらち, あるものは近似によるものだ し, 他のものは逆解法で得た解を境界条件として順解 法に代大し, その解が再び逆解法の境界条件に一致す る温度関数を与える点でより㛜格である。特に，それ が Buggraff 法3)によって得られた解であれば正しい。 文献2)には，そのような解が熱伝達問題順解法に対 応する解から見つけられることが示されている。その 上，Buggraff 法を使えば得られる解が 比較的簡単な 方法で記述できる利点がある。

本論文では，実際に興味深い二次元空間座標で表わ した極低温液体の逆熱伝達問題の解法について，一般 化一次元 Buggraff 法を使って求めている。

\section{問題の提起}

二次元非定常熱伝達の解析を無限長二重円筒を例と して行なってみる。ただし, この円筒は一定の熱物理

*電子技術総合研究所高エネルギ研究室
特性をもつ二種類の材質でできており，その表面は理 想的な熱接触が成立すると仮定する。実際に, この例 は極低温流体が熱伝達率の低いコーティングを施した パイプラインを冷却する場合に応用できる。

$$
\begin{aligned}
& \frac{\partial^{2} T}{\partial r^{2}}+\frac{1}{r} \frac{\partial T}{\partial r}+\frac{\partial^{2} T}{\partial z^{2}}=\frac{1}{a_{\mathrm{i}}} \frac{\partial T}{\partial \tau} \\
& \text { ここに } a_{\mathrm{i}}=a_{0} \text { at } 0 \leq r \leq r_{1} \\
& a_{\mathrm{i}}=a_{2} \text { at } r_{1} \leq r \leq r_{2}
\end{aligned}
$$$$
\text { 初期条件は } T(r, z, 0)=T \text { may }(r, z)
$$

であり，連続円筒軸の境界条件は次の通りである。

$$
\frac{\partial T}{\partial r}(0, z, \tau)=0
$$

内部円筒と外部冈筒の内表面に関する境界条件は次 の通りである。

$$
\begin{aligned}
& T\left(r_{1}, z, \tau\right)=T_{1}(z, \tau) \\
& -\lambda_{0} \frac{\partial T}{\partial r}\left(r_{1}-0, z, \tau\right)=-\lambda_{2} \frac{\partial T}{\partial r}\left(r_{1}+0, z, \tau\right) \\
& =q_{1}(z, \tau)
\end{aligned}
$$

ここに一般的には, $T_{1}(z, \tau)$ は既知関数であり, $q_{1}$ $(z, \tau)$ は未知関数である。 
境界条件は， $z \rightarrow \pm \infty$ で

$$
\frac{\partial T}{\partial z}(r, \pm \infty, \tau)=0
$$

である。この問題を解くにあたり, 温度分布 $T(r, z$, て)及び特に，二重円筒の外側表面上の温度と熱流束の 関係を決めておく必要がある。

$$
\begin{aligned}
& T_{2}(z, \tau)=T\left(r_{2}, z, \tau\right) \\
& q_{2}(z, \tau)=-\lambda_{2} \frac{\partial T}{\partial r}\left(r_{2}, z, \tau\right)
\end{aligned}
$$

実際には, 関数 (4)式を測定して求め, これを基に (7)，（8)式を変形して解を求める。それゆえ， $T_{2}$ $(z, \tau)$ と $q_{2}(z, \tau)$ を $T_{1}(z, \tau)$ によって表わす必要があ る。

(1) ～(8) 式は領域が $0 \leq r \leq r_{1}$ では，順解法によ り正しく求まるが，領域が $r_{1} \leq r \leq r_{2}$ では順解法では 正しく解を求めることができず，逆解法を行なう必要 がある。しかし，両領域においても Buggraff 法の二 次元修正解法を使らのが適当である。

\section{領域 $0 \leq r \leq r_{1}$ の解}

解析を行ならにあたり, 次なる無次元変数を導入す る。

$$
R=\frac{r}{r_{1}}, \quad z=\frac{z}{r_{1}}, \quad t=\frac{a_{\mathrm{j}} \tau}{r_{\mathrm{i}}^{2}}, \quad \theta=\frac{T-T_{\min }}{T_{\max }-T_{\min }}
$$

ここに $T_{\max }, T_{\min }$ 等は特性温度 (初期, 最終温 度とか, 中間温度) である。

方程式 (1) 式と境界条件 (3)，（4)式は次のように なる。

$$
\begin{aligned}
& \frac{\partial^{2} \theta}{\partial R^{2}}+\frac{1}{R} \frac{\partial \theta}{\partial R}+\frac{\partial^{2} \theta}{\partial z^{2}}=\frac{\partial \theta}{\partial t} \\
& \frac{\partial \theta}{\partial R}(0, z, t)=0 \\
& \theta(1, z, t)=\theta,(z, t)
\end{aligned}
$$

この解は次のような形で表わせる。

$$
\theta(R, z, t)=\sum_{\mathrm{m}=0}^{\infty} \sum_{\mathrm{n}=0}^{\infty} \varphi_{\mathrm{mn}}(R) \frac{\partial^{\mathrm{m}+\mathrm{n}} \theta_{1}}{\partial z^{\mathrm{m}} \partial t^{\mathrm{n}}}
$$

ここで $\varphi_{\mathrm{mn}}(R)$ は決めるべき未知関数である。

解 (13) 式は自動的に条件 (6), (2) 式を満たす。し かし, $\tau \rightarrow 0, z \rightarrow \pm \infty$ では級数列 (13) 式の一致性は低 下するはずである。この現象は逆解法では普通である。 (13) 式を (10) 式及び境界条件式 (11)，(12) 式に代入し て, 同じ微係数項で整理すると, 次に示す $\varphi_{\mathrm{mn}}(R)$ 方 程式の集合を得る。

$$
\varphi^{\prime \prime}{ }_{00}+\frac{1}{R} \varphi^{\prime}{ }_{00}=0
$$

$$
\begin{aligned}
& \varphi_{10}^{\prime \prime}+\frac{1}{R} \varphi^{\prime}{ }_{10}=0 \\
& \varphi^{\prime \prime}{ }_{0 \mathrm{n}}+\frac{1}{R} \varphi_{0 \mathrm{n}}^{\prime}=\varphi_{0, \mathrm{n}-1}(n \geqq 1) \\
& \varphi^{\prime \prime}{ }_{1 \mathrm{n}}+\frac{1}{R} \varphi_{1 \mathrm{n}}^{\prime}=\varphi_{1, \mathrm{n}-1}(n \geq 1) \\
& \varphi^{\prime \prime}{ }_{\mathrm{mo}}+\frac{1}{R} \varphi_{\mathrm{mo}}^{\prime}=-\varphi_{\mathrm{m}-2,0}(m \geq 2) \\
& \varphi^{\prime \prime} \mathrm{mn}+\frac{1}{R} \varphi_{\mathrm{mn}}^{\prime}=\varphi_{\mathrm{m}, \mathrm{n}-1}-\varphi_{\mathrm{m}-2, \mathrm{n}}(m \geq 2, \\
& n \geq 1)
\end{aligned}
$$

境界条件は

$$
\begin{aligned}
& \varphi_{00}(1)=1 \\
& \varphi_{\mathrm{mn}}(1)=0 \\
& \varphi^{\prime} \mathrm{mn}(0)=0
\end{aligned}
$$

である。

こうして, $m, n$ を増加させ $(14) \sim(19)$ 式を解くと， 次式を得る。

$$
\varphi_{\mathrm{mn}}(R)=\left\{\begin{array}{l}
0 \quad \text { at } m=2 K-1 \\
(-1) \mathrm{K} \frac{(K+n) !}{K ! n !} \varphi_{\theta \mathrm{K}+\mathrm{n}}(R) \text { at } m=2 K
\end{array}\right.
$$

第一関数 $\varphi_{\mathrm{op}}(R)$ の一般的な形は次のように表わせ る。

$$
\varphi_{\mathrm{op}}(R)=\frac{1}{\left(2^{\mathrm{p} P !}\right)^{2}} \sum_{\mathrm{s}=0}^{\mathrm{p}} r_{\mathrm{ps}} R^{2 \mathrm{~s}}
$$

(24)を(16)式に代入して，同じRの累乗項の係数を等 しくすると, 次に示す式を得る。

$$
r_{\mathrm{ps}}=r_{\mathrm{p}-1, \mathrm{~s}-1}\left(\frac{P}{S}\right)^{2}, \quad(1 \leq S \leq P)
$$

(21)式の条件加，

$$
r_{\mathrm{po}}=-\sum_{\mathrm{s}=1}^{\mathrm{p}} r_{\mathrm{ps}}
$$

となり，(20)，(22)式を考慮して (14) 式から

$$
\varphi_{\infty}(R)=1
$$

を得る。これより $r_{00}=1$ となる。

こうして， $S=1,2, \cdots P, \overline{\mathrm{o}}$ に対し $P$ を連続的に 増すと, 係数 $r_{\mathrm{ps}}$ は (25)，(26)式から求まる。

(23)，(24)式を考慮すると， $0 \leq R \leq 1$ の領域におけ る温度は次のような形となる。

$$
\begin{gathered}
\theta(R, z, t)=\sum_{\mathrm{k}=0}^{\infty} \sum_{\mathrm{n}=0}^{\infty} \frac{(-1) \mathrm{s}_{\mathrm{S}=0}^{\mathrm{K}+\mathrm{n}} r_{\mathrm{k}+\mathrm{n}, 2} R^{2 \mathrm{~s}}}{K ! n !(K+n) ! 2^{2(\mathrm{k}+\mathrm{n})}} \\
\frac{\partial^{2 \mathrm{k}+\mathrm{n} \theta_{\mathrm{l}}}}{\partial z^{2 \mathrm{k}} \partial t^{\mathrm{n}}} \\
r=r_{1} \text { における表面熱流束は } \\
q_{1}(z, t)=-\lambda_{\theta}\left(\frac{\partial T}{\partial r}\right)_{\mathrm{r}=\mathrm{r}_{1}}=-\lambda_{\theta}\left(T_{\max }-T_{\min }\right) \\
\frac{1}{r_{1}}\left(\frac{\partial \theta}{\partial R}\right)_{\mathrm{R}=1}
\end{gathered}
$$


また，有次元系で表わせば

$$
\begin{aligned}
& q_{1}(z, \tau)=\frac{\lambda_{0}}{r_{\mathrm{i}}} \sum_{\mathrm{k}=0}^{\infty} \sum_{\mathrm{n}=0}^{\infty} \frac{(-1)^{\mathrm{k}+1}}{K ! n !} G_{\mathrm{k}+\mathrm{n}} \frac{r_{1}^{2 \mathrm{k}+\mathrm{n}}}{a_{\mathrm{i}}^{\mathrm{n}}} . \\
& \frac{\partial^{2 \mathrm{k}+\mathrm{n}} T_{1}}{\partial z^{2 \mathrm{k}} \partial \tau^{\mathrm{n}}}
\end{aligned}
$$

となる。ここで

$$
G_{\mathrm{p}}=\frac{1}{P ! 2^{2 \mathrm{p}-1}} \sum_{\mathrm{s}=0}^{\mathrm{p}} s r_{\mathrm{ps}}
$$

である $\left(G_{0}=0, G_{1}=\frac{1}{2}, G_{2}=-\frac{1}{8}, G_{3}=\frac{1}{16} \cdots\right.$ etc $)$ 。

\section{領域 $r_{1} \leq r \leq r_{2}$ の解}

中空円筒 $\left(r_{1} \leq r \leq r_{2}\right)$ の境界条件 (5) 式は (30) 式で 表わせる。解析にあたり, 次の無次元変数を領域 $r_{1} \leq$ $r \leq r_{2}$ に導入する。

$$
\begin{gathered}
R=\frac{r}{r_{1}}, \quad z=\frac{z}{r_{1}}, \quad t=\frac{a_{2} \tau}{r_{1}{ }^{2}}, \quad \theta=\frac{T-T_{\min }}{T_{\max }-T_{\min }}, \\
Q=\frac{q r_{1}}{\lambda_{2}\left(T_{\max }-T_{\min }\right)}
\end{gathered}
$$

方程式 ( 1 ) 式は (10)式の形になり, 境界条件は次の ようになる。

$$
\begin{aligned}
& \theta(1, z, t)=\theta_{1}(z, t) \\
& \left(\frac{\partial \theta}{\partial R}\right)_{\mathrm{R}=1}=\theta_{3}(z, t)
\end{aligned}
$$

解は初期条件 (2) 式及び境界条件 (6) 式を自動的に 満たす形として求められる。

$$
\begin{gathered}
\theta(R, z, t)=\sum_{\mathrm{m}=0}^{\infty} \sum_{\mathrm{n}=0}^{\infty} f_{\mathrm{mn}}(R) \frac{\partial^{\mathrm{m}+\mathrm{n}} \theta_{1}}{\partial z^{\mathrm{m}} \partial t^{\mathrm{n}}}-\sum_{\mathrm{m}=0}^{\infty} \sum_{\mathrm{n}=0}^{\infty} \\
g_{\mathrm{mn}}(R) \frac{\partial^{\mathrm{m}+\mathrm{n}} Q}{\partial z^{\mathrm{n}} \partial t^{\mathrm{n}}}
\end{gathered}
$$

$f_{\mathrm{mn}}(R)$ 及び $g_{\mathrm{mn}}(R)$ は求めるべき未知関数である。 (35) 式を(10) 式に代入して微分項係数を等しく置くと $f_{\mathrm{mr}}(R), g_{\mathrm{mn}}(R)$ の微分方程式の集合を得る。これ は $(14) \sim(19)$ 式に示した $\varphi_{\mathrm{mn}}(R)$ と等価である。

(35)式を境界条件 (33)，(34)式に代入して，同じ微 分係数を等しく置くと次示を得る。

$$
\begin{aligned}
& f_{00}(1)=1 \\
& f_{\mathrm{mn}}(1)=0 ; \quad(m+n>0) \\
& f^{\prime}{ }_{\mathrm{mn}}(1)=0 ; \quad(m \geq 0, \quad n \geq 0) \\
& g_{\mathrm{mn}}(1)=0 ; \quad(m \geq 0, n \geq 0) \\
& g^{\prime}{ }_{00}(1)=1 \\
& g^{\prime}{ }_{\mathrm{mn}}(1)=0(m+n>0)
\end{aligned}
$$

$(36) \sim(39)$ 式で示した $f_{\mathrm{mn}}(R), g_{\mathrm{mn}}(R)$ に対して $(14) \sim(19)$ 式の場合と同様な計算を行なうと, $f_{\mathrm{mn}}$ $(R), g_{\mathrm{mn}}(R)$ に対しても，(23) 式が成立することが わかる。
これらの主関数 $f_{\mathrm{op}}(R), g_{\mathrm{op}}(R)$ を計算すると, 次 のような一般形式になるはずである。

$$
\begin{aligned}
& f_{\mathrm{op}}(R)=\frac{1}{(2 \mathrm{p} P !)^{2}} \sum_{\mathrm{s}=0}^{\mathrm{p}}\left(\varepsilon_{\mathrm{ps}} R^{2 \mathrm{~s}}+\zeta_{\mathrm{ps}} R^{2 \mathrm{~s}} \ln R\right) \\
& g_{\mathrm{op}}(R)=\frac{1}{\left(2 \mathrm{p} P^{\prime}\right)^{2}} \sum_{\mathrm{s}=0}^{\mathrm{p}}\left(æ_{\mathrm{ps}} R^{2 \mathrm{~s}}+\varphi_{\mathrm{ps}} R^{2 \mathrm{~s}} \ln R\right)
\end{aligned}
$$

(16)式の場合と同様に，(42) 式 $f_{\mathrm{op}}(R)$ に代入して, 両辺の同じ $R$ 項係数を等しいとおくと, 係数 $\varepsilon_{\mathrm{ps}}$, $\zeta_{\mathrm{ps}}$ に関して次のような式を得る。

$$
\begin{aligned}
& \varepsilon_{\mathrm{ps}}=\varepsilon_{\mathrm{p}-1, \mathrm{~s}-1} \frac{P^{2}}{S^{2}}-\zeta_{\mathrm{p}-\mathrm{i}}, \mathrm{s}-1 \frac{P_{2}}{S_{\mathrm{s}}}(1 \leq S \leq P) \\
& \varepsilon_{\mathrm{po}}=-\sum_{\mathrm{s}=1}^{\mathrm{p}} \varepsilon_{\mathrm{ps}} \quad(P \geq 1) \\
& \zeta_{\mathrm{ps}}=\zeta_{\mathrm{p}-1, \mathrm{~s}-1} \frac{P^{2}}{S^{2}}(1 \leq S \leq P) \\
& \zeta_{\mathrm{po}}=\sum_{\mathrm{s}=1}^{\mathrm{p}} \zeta_{\mathrm{ps}}-2 P^{2} \sum_{\mathrm{s}=1}^{\mathrm{p}} \frac{\varepsilon_{\mathrm{p}-1}, \mathrm{~s}-1}{S}(P \geq 1)
\end{aligned}
$$

(36)，(38)式を考慮すると $f_{00}(R)$ に関して次式を得 る。これは $(14)$ 式の場合と同じである。

$$
f_{00}(R)=1
$$

(48)式から $\varepsilon_{00}=1, \zeta_{00}=0$ となる。

こうして, 係数 $\varepsilon_{\mathrm{ps}}, \varphi_{\mathrm{ps}}$ は $S=1,2,3, \cdots P, 0$ の 各值に対し $P$ を増加させ $(44) \sim(47)$ 式から求めること ができる。また，(43)式の係数 ※ps は (44)〜 (47) 式

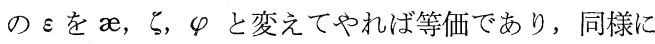
して求めることができる。

(14)式と同様に，(39)，(40)式を考慮すると $g_{00}(R)$ が求まる。

$$
g_{00}(R)=\ln R
$$

(49)式から $æ_{00}=0, \varphi_{00}=1$ となり続く係数は (44) 〜 (47) 式に示したと同様な式を使って求めることがで きる。

\section{最終的な無次元系で表わした二重円筒の外層温度} (内表面温度, 熱流束密度変化が既知の場合の中空円 筒では外層温度）は次のように表わせる。

$$
\begin{aligned}
& \theta(R, z, t)=\sum_{\mathrm{k}=0}^{\infty} \sum_{\mathrm{n}=0}^{\infty} \frac{(-1)^{\mathrm{k}}}{K ! n !} \frac{\sum_{\mathrm{k}=0}^{\mathrm{n}+\mathrm{k}}\left(\varepsilon_{\mathrm{k}+\mathrm{n}, \mathrm{s}} R^{2 \mathrm{~s}}+\zeta_{\mathrm{k}+\mathrm{n}, \mathrm{s}}\right.}{2^{2(\mathrm{k}+\mathrm{n})}(n+K) !}
\end{aligned}
$$

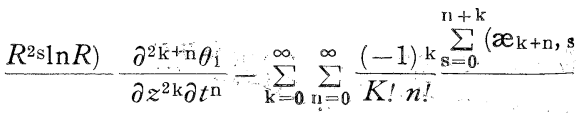

$$
\begin{aligned}
& \frac{\left.R^{2 \mathrm{~s}}+\varphi_{\mathrm{k}+\mathrm{n}, \mathrm{s}} R^{2 \mathrm{~s}} \ln R\right)}{2^{2(\mathrm{k}+\mathrm{n})}(K+n) !} \frac{\partial^{2 \mathrm{k}+\mathrm{n}} Q_{1}}{\partial z^{2 \mathrm{k}} \mathrm{n}^{\mathrm{n}}}
\end{aligned}
$$




\section{薄い外部層の場合}

二重円筒の外部層が薄い場合には，(50) 式は計算上 都合の良い形ではない。なぜならば， $(50)$ 式を外部層 が薄い場合に適用すると, 大容積内の状態の微差を計 算することになるからである。それゆえ，薄い中空円 筒に対してはより都合の良い式を見つける必要がある (ここで $\left.b=r_{2}-r_{1} \ll r_{i}\right) 。$

新しい座標として $x=r-r_{t}$ を導入する。これは壁 厚さによる温度分布変化を特性づける。対応する無次 元座標は

$$
x=\frac{x}{r_{1}}=R-1
$$

であり， $x$ は $0 \leq x \leq B$ の領域では $x \leq 1$ である。こ こで $B$ は $b / \tau_{1}$ を示し，無次元壁厚を意味する。

この場合の温度分布は次のような形で表わせる。

$$
\begin{gathered}
\theta(x, z, t)=\sum_{\mathrm{m}=0}^{\infty} \sum_{n=0}^{\infty} f_{\mathrm{mn}}(x) \frac{\partial^{\mathrm{m}+\mathrm{n}} \theta_{1}}{\partial z^{\mathrm{m}} \partial t^{\mathrm{n}}}-\sum_{\mathrm{m}=0}^{\infty} \sum_{\mathrm{n}=0}^{\infty} \\
g_{\mathrm{m} n}(x) \frac{\partial^{\mathrm{m}+\mathrm{n}} Q_{1}}{\partial z^{\mathrm{m}} \partial t^{\mathrm{n}}}
\end{gathered}
$$

(35)，(52) を比較すると $f_{\mathrm{mn}}(x), g_{\mathrm{mn}}(x)$ と $f_{\mathrm{mn}}(R)$, $g_{\mathrm{mn}}(R)$ とは同じ形であり, 特に, $(16)$ 式と $(23)$ 式の 形は全く同じになる。

その上, (48), (49)式から次式が成立する。

$$
\begin{aligned}
& f_{00}(x)=1 \\
& g_{00}(x)=\ln (1+x)=\sum_{s=1}^{\infty} \frac{(-1)^{s^{-1}}}{S} x^{s}
\end{aligned}
$$

$R=1+x$ を (42)，(43) 式に代入して, $\ln (1+x)$ を 級数展開すると， $x$ の無限多項式が得られる。 $x \ll 1$ の場合には, $f_{\mathrm{op}}(x), g_{\mathrm{op}}(x)$ は次のようになる。

$$
\begin{aligned}
& f_{\mathrm{op}}(x)=\sum_{\mathrm{s}=0}^{\infty} h_{\mathrm{ps}} x^{\mathrm{s}} \\
& g_{\mathrm{op}}(x)=\sum_{\mathrm{s}=0}^{\infty} l_{\mathrm{ps}} x^{2}
\end{aligned}
$$

(53), (55) 式加ら

$$
h_{\mathrm{Oo}}=1, \quad h_{\mathrm{OS}}=0 \quad(S \geq 1)
$$

が得られ，(54)，(56)式から

$$
l_{\mathrm{oo}}=0 ; l_{\mathrm{os}}=(-1)^{\mathrm{s}-1} / S(S \geq 1)
$$

が得られる。(55), (56) 式を(16) 式と同じ形の微分方 程式に代入し, また境界条件 (36) (41) 式に代入する と, 次式を得る。ただし， $R=1$ で $x=0$ であること を注意する必要がある。

$$
\begin{aligned}
& h_{\mathrm{po}}=h_{\mathrm{p} 1}=h_{\mathrm{p} 2}=0 \quad(P \geq 1) \\
& h_{\mathrm{ps}}=\frac{h_{\mathrm{p}-1, \mathrm{~s}-2}+h_{\mathrm{p}-1, \mathrm{~s}-3}-(S-1)^{2} h_{\mathrm{p}, \mathrm{s}-1}}{S(S-1)} \\
& l_{\mathrm{op}}=l_{\mathrm{ps}}=0 \quad(P \geq 1)
\end{aligned}
$$

Table 1 Coefficients hps

\begin{tabular}{|c|ccccccccc|}
\hline $\mathrm{p}$ & 0 & 1 & 2 & 3 & 4 & 5 & 6 & 7 & 8 \\
\hline 0 & 1 & 0 & 0 & 0 & 0 & 0 & 0 & 0 & 0 \\
\hline 1 & 0 & 0 & $\frac{1}{2}$ & $-\frac{1}{6}$ & $\frac{1}{8}$ & $-\frac{1}{10}$ & $\frac{1}{12}-\frac{1}{14}$ & $\frac{1}{16}$ \\
\hline 2 & 0 & 0 & 0 & 0 & $\frac{1}{24}-\frac{1}{60}$ & $\frac{1}{80}-\frac{17}{1680}$ & $\frac{23}{2688}$ \\
\hline 3 & 0 & 0 & 0 & 0 & 0 & 0 & $\frac{1}{720}-\frac{1}{1680}$ & $\frac{1}{2240}$ \\
\hline 4 & 0 & 0 & 0 & 0 & 0 & 0 & 0 & 0 & $\frac{1}{40320}$ \\
\hline
\end{tabular}

Table 2 Coefficients $1 \mathrm{ps}$

\begin{tabular}{|c|cccccccc|}
\hline$p^{-s}$ & 0 & 1 & 2 & 3 & 4 & 5 & 6 & 7 \\
\hline 0 & 0 & 1 & $-\frac{1}{2}$ & $\frac{1}{3}$ & $-\frac{1}{4}$ & $\frac{1}{5}$ & $-\frac{1}{6}$ & $\frac{1}{7}$ \\
\hline 1 & 0 & 0 & 0 & $\frac{1}{6}$ & $-\frac{1}{12}$ & $\frac{7}{120}$ & $-\frac{11}{240}$ & $\frac{4}{105}$ \\
\hline 2 & 0 & 0 & 0 & 0 & 0 & $\frac{1}{120}$ & $-\frac{1}{240}$ & $\frac{1}{336}$ \\
\hline 3 & 0 & 0 & 0 & 0 & 0 & 0 & 0 & $\frac{1}{5040}$ \\
\hline
\end{tabular}

$$
\begin{aligned}
& l_{\mathrm{p} 2}=\left(l_{\mathrm{p}-1,0}-l_{\mathrm{p} 1}\right) / 2 \\
& l_{\mathrm{ps}}=\frac{l_{\mathrm{p}-1, \mathrm{~s}-2}+l_{\mathrm{p}-1, \mathrm{~s}-3}-(S-1)^{2} l_{\mathrm{p}, \mathrm{s}-1}}{S(S-1)}(S \geq 3)
\end{aligned}
$$

これら係数 $h_{\mathrm{ps}}, l_{\mathrm{ps}}$ の值を Table 1,2 に示した。 表に示したデータを一般化する上で，(60)，(63)式 に示した式より便利な形がある。つまり(55)，(56)式 は次のような式で表わすことができる。

$$
\begin{gathered}
f_{\mathrm{op}}(x)=\frac{x^{2} P}{2 P !}\left[1-\frac{P}{2 P+1} x+\frac{3}{4} \frac{P}{2 P+1} x^{2}-\frac{1}{4}\right. \\
\frac{(5 P+7)}{(2 P+1)(2 P+3)} x^{3}+\frac{5}{32} \frac{P(7 P+9)}{(2 P+1)(2 P+3)} \\
\left.x^{4} \ldots \ldots\right) \\
g_{\mathrm{op}}(x)=\frac{x^{2} \mathrm{P}+1}{(2 P+1) !}\left[1-\frac{1}{2} x-\frac{1}{4} \frac{3 P+Y}{2 P+3} x^{2}\right. \\
\left.-\frac{1}{8} \frac{5 P+6}{2 P+3} x^{3}+\cdots \cdots\right)
\end{gathered}
$$

薄い中空円筒の無次元系で示した温度，あるいは二 重円筒の薄い外層内のそれは次のようになる。

$$
\begin{aligned}
& \theta(x, z, t)=\sum_{\mathrm{k}=0}^{\infty} \sum_{\mathrm{n}=0}^{\infty}(-1) \mathrm{k} \frac{(K+n) !}{K ! n !} f_{0, \mathrm{k}+\mathrm{n}}(x) \cdot \\
& \frac{\partial^{2} \mathrm{k}+\mathrm{n} \theta_{1}}{\partial z^{2} \mathrm{k} \partial t^{\mathrm{n}}}-\sum_{\mathrm{k}=0}^{\infty} \sum_{\mathrm{n}=0}^{\infty}(-1) \mathrm{k} \frac{(K+n) !}{K ! n !} g_{0, \mathrm{k}+\mathrm{n}}(x) \\
& \frac{\partial^{2 \mathrm{k}+\mathrm{n}} Q_{1}}{\partial z^{2} \mathrm{k} \partial t^{\mathrm{n}}}
\end{aligned}
$$

無次元系で示した外表面温度は次の通りである。

$$
\theta_{2}(z, t)=\theta(B, z, t)=\sum_{\mathrm{k}=0}^{\infty} \sum_{\mathrm{n}=0}^{\infty} \frac{(-1)^{\mathrm{k}}}{K ! n !}\left(D_{\mathrm{k}+\mathrm{n}} \cdot\right.
$$

Vol. 14 No. 2 (1979) 


$$
\left.\frac{\partial^{2 \mathrm{k}+\mathrm{n}} \theta_{1}}{\partial z^{2 \mathrm{k}} \partial t^{\mathrm{n}}}-H_{\mathrm{k}+\mathrm{n}} \frac{\partial^{2 \mathrm{k}+\mathrm{n}} Q_{1}}{\partial z^{2 \mathrm{k}} \partial t^{\mathrm{n}}}\right)
$$

ここで

$$
\begin{aligned}
D_{\mathrm{p}} & =\frac{P !}{(2 P) !} B^{2} \mathrm{P}^{\zeta}\left(1-\frac{P}{2 P+1} B+\frac{3}{4} \frac{P}{2 P+1} B^{2}\right. \\
& \left.-\frac{1}{4} \frac{P(5 P+7)}{(2 P+1)(2 P+3)} B^{3}+\cdots \cdots\right) \\
H_{\mathrm{p}} & =\frac{P !}{(2 P+1) !} B^{2 \mathrm{P}+1}\left[1-\frac{1}{2} B+\frac{1}{4} \frac{3 P+4}{2 P+3} B^{2}\right. \\
& \left.-\frac{1}{8} \frac{5 P+6}{2 P+3} B^{3}+\cdots \cdots\right)
\end{aligned}
$$

また, 無次元系で表わした円筒外表面の熱流束は次 のよらになる。

$$
\begin{aligned}
& \theta_{2}(z, t)=-\frac{\partial \theta}{\partial R}(B, z, t)=\sum_{\mathrm{k}=0}^{\infty} \sum_{n=0}^{\infty} \frac{(-1)^{\mathrm{k}+1}}{K ! n !} \cdot \\
& \left(E_{\mathrm{k}+\mathrm{n}} \frac{\partial^{2 \mathrm{k}+\mathrm{n}} \theta_{1}}{\partial z^{2 \mathrm{k}} \partial t^{\mathrm{n}}}-L_{\mathrm{k}+\mathrm{n}} \frac{\partial^{2 \mathrm{k}+\mathrm{n}} Q_{!}}{\partial z^{2 \mathrm{k}} \partial t^{\mathrm{n}}}\right) \\
& \text { ここに } \\
& E_{0}=0 \\
& E_{\mathrm{p}}=(P !) f^{\prime}{ }_{\mathrm{op}}(B)=\frac{P !}{(2 P-1) !} B^{\circ} \mathrm{P}^{-1}\left(1-\frac{1}{2} B\right. \\
& \left.+\frac{3}{4} \frac{P+1}{2 P+1} B^{2}-\frac{1}{8} \frac{5 P+7}{2 P+1} B^{3}+\cdots \cdots\right) \\
& L_{\mathrm{p}}=(P !) g^{\prime}{ }_{\mathrm{op}}(B)=\frac{P !}{(2 P !)} B^{2 \mathrm{p}}\left(1-\frac{P+1}{2 P+1} B\right. \\
& +\frac{1}{4} \frac{3 P+4}{2 P+1} B^{2}-\frac{1}{4} \frac{(P+2)(5 P+6)}{(2 P+1)(2 P+3)} \\
& \left.B^{3}+\cdots \cdots\right)
\end{aligned}
$$

(66)～(72) 式は中空円筒の場合にも適用できる。この 場合には二物体間接触面での熱流束が温度によって記 述できれば，(30)式を考慮すると変形が可能である。

(32)式を考慮して

$$
\begin{gathered}
Q_{1}(z, t)=\frac{q_{\mathrm{i}} r_{i}}{\lambda_{2}\left(T_{\max }-T_{\min }\right)}=\frac{1}{A_{\mathrm{i}}} \sum_{0}^{\infty} \sum_{\mathrm{j}=0}^{\infty} \frac{(-1)}{i ! J !} . \\
G_{\mathrm{i}+\mathrm{j}} A \mathrm{j} \frac{\partial^{2 \mathrm{i}+\mathrm{j} \theta_{1}}}{\partial z^{2 \mathrm{i}} \partial t^{\mathrm{j}}}
\end{gathered}
$$

ここに $A=\lambda_{2} / \lambda_{0}, \quad A=a_{2} / a_{0}$

(73)式を (67)，(70)式に代入すると次式を得る。

$$
\begin{gathered}
\theta_{2}(z, t)=\sum_{\mathrm{k}=0}^{\infty} \sum_{\mathrm{n}=0}^{\infty} \frac{(-1)^{\mathrm{k}}}{K ! n !}\left(D_{\mathrm{k}+\mathrm{n}} \frac{\partial^{2} \mathrm{k}+\mathrm{m} \theta_{1}}{\partial z^{2 \mathrm{k}} \partial t^{\mathrm{n}}}\right. \\
\left.-\frac{H_{\mathrm{k}+\mathrm{n}}}{A} \sum_{\mathrm{i}=0}^{\infty} \sum_{\mathrm{j}=0}^{\infty} \frac{(-1)^{\mathrm{i}+\mathrm{j}}}{i ! j !} G_{\mathrm{i}+\mathrm{j}} A \mathrm{j} \frac{\partial^{2 \mathrm{k}+\mathrm{n}+2 \mathrm{i}+\mathrm{j}} \theta_{\mathrm{i}}}{\partial z^{2 \mathrm{k}+2 \mathrm{i}} \partial t^{\mathrm{n}+\mathrm{j}}}\right] \\
\theta(z, t)=\sum_{\mathrm{k}=0}^{\infty} \sum_{\mathrm{n}=0}^{\infty} \frac{(-1)^{\mathrm{k}+1}}{K ! n !}\left[E_{\mathrm{k}+\mathrm{n}} \frac{\partial^{2 \mathrm{k}+\mathrm{n}} \theta_{1}}{\partial z^{2 \mathrm{k}} \partial t^{\mathrm{n}}}\right. \\
\left.-\frac{L_{\mathrm{k}+\mathrm{n}}}{A} \sum_{\mathrm{i}=0}^{\infty} \sum_{\mathrm{j}=0}^{\infty} \frac{(-1)^{\mathrm{i}+\mathrm{j}}}{i ! j !} G_{\mathrm{i}+\mathrm{j}} A \mathrm{j} \frac{\partial^{2 \mathrm{k}+\mathrm{n}+2 \mathrm{i}+\mathrm{j} \theta_{1}}}{\partial z^{2} \mathrm{k}+2 \mathrm{n} \partial t^{\mathrm{n}+\mathrm{j}}}\right]
\end{gathered}
$$

実際に計算する場合には，(74)，(75)式を次のよう に表わした方が便利である。

$$
\begin{aligned}
& \theta_{2}(z, t)=\sum_{\mathrm{p}=0}^{\infty} \sum_{\mathrm{s}=0}^{\infty} M_{\mathrm{ps}} \frac{\partial^{2 \mathrm{P}+\mathrm{s}} \theta_{i}}{\partial z^{2 \mathrm{P} \partial t}} \\
& \theta_{2}(z, t)=\sum_{\mathrm{p}=0}^{\infty} \sum_{\mathrm{s}=0}^{\infty} N_{\mathrm{ps}} \frac{\partial^{2 \mathrm{P}+\mathrm{s}} \theta_{1}}{\partial z^{2 \mathrm{P} \partial} t^{\mathrm{s}}}
\end{aligned}
$$

ここに

$$
\begin{aligned}
& M_{\mathrm{Ps}}=(-1) \mathrm{p}\left[\frac{D_{\mathrm{p}+\mathrm{s}}}{P ! S !}+\sum_{\Lambda} \sum_{1=0}^{\mathrm{P}} \sum_{\mathrm{n}=0}^{\mathrm{s}}\right. \\
& \left.\frac{H_{\mathrm{p}-1+\mathrm{s}-\mathrm{m}} G_{1+\mathrm{m}} A \mathrm{~m}}{l ! m !(P-l) !(S-m) !}\right] \\
& N_{\mathrm{ps}}=(-1)^{\mathrm{p}+1}\left[\frac{E_{\mathrm{p}+\mathrm{s}}}{P ! S !}+\frac{1}{\Lambda} \sum_{1=0}^{p} \sum_{\mathrm{m}=0}^{:}\right. \\
& \left.\frac{L_{\mathrm{p}-1+\mathrm{s}-\mathrm{m}} Q_{1+\mathrm{m}} A \mathrm{~m}}{l ! m !(P-l) !(S-m) !}\right]
\end{aligned}
$$

二重円筒の幾何学的構造とか材質の温度物理特性が 既知であれば，係数 $G$ は (31) 式から，D， H, E , L は $(68) \sim(72)$ 式から求まるので, $M_{\mathrm{ps}}, N_{\mathrm{ps}}$ を知るこ とができる。このように，係数 $M_{\mathrm{ps}} ， N_{\mathrm{ps}}$ を一度決 めておけば, テストデータを求めると簡単な $(76),(77)$ 式を使って， $\theta_{1}(z, t)$ を求めることができる。

同様にして，三重あるいは多重円筒の場合にもこれ まで述べた中空円筒からなる二重円筒計算法を適用す ることができる。この場合は (76)，(77) 式は次の円筒 層の境界条件となる。実際に解く場合には，まず状態 を次元形式で表現し，次に，(36)式以後で述べたよう な無次元形式に変換し, これら変換された变数を使っ て, 次の円筒層温度分布を $(50),(66)$ 式により求める のである。

\section{円筒軸に沿って等温線が一定速度で移動して いる場合の温度分布}

実験から関数 $T_{1}(z, T)$ を $\approx, T$ の無限個の関係で 示すことは不可能である。実際にはレコーダで測定で きる有限個の $T_{1}(z)$ を知るだけである。従って，一般 的には $z$ 軸方向の微分計算は大きな誤差を生じさせて しまう。しかし，実際に重要である特殊な場合におい ては，温度分布にある規則性が存在する。この規則性 がある場合には関数 $T_{1}(z)$ の情報不足はカバーされる ことになる。

実験データを正確に知る上で，最も都合の良い場合 とは, 曲線 $T_{1}\left(z_{1}, \tau\right)$ が時間軸に沼って等間隔に配置 されている場合であり, 同時刻において等距離 $\Delta z=$ $z_{\mathrm{i}+1}-z_{\mathrm{i}}$ に等時間間隔 $\Delta \tau=\tau_{\mathfrak{i}+!}-\tau_{\mathrm{i}}$ が対応している 場合である。つまりこの場合には， $T_{1}\left(z_{1}, \tau_{1}\right)=T_{1}$ ・ 
$\left(z_{\mathrm{j}+1}, \tau_{\mathrm{i}+1}\right)$ となる。

このことは温度 $T_{1}$ の各々の值が $z$ 軸に沿って一定 速度 $V$ で移動していることを意味する。

$$
V=\frac{z_{\mathrm{i}+1}-z_{\mathrm{i}}}{\tau_{\mathrm{i}+1}-\tau_{\mathrm{i}}}
$$

この場合, (81) 式の座標点上の温度は $T_{1}$ となる。

$$
z=z_{1}+V\left(\tau-\tau_{1}\right)
$$

そして，この温度は時刻 $\tau_{\mathrm{i}}$ で観測される $z_{\mathrm{i}}$ 点のま た時刻 $\tau_{i+1}$ で観測される $z_{\mathrm{i}+1}$ 点の温度でもある。

微分要素 $d z=V d \tau$ 索使って座標軸に沿って微分す 万と，時間街分は次のようになる。

$$
\left(\frac{\partial^{2 \mathrm{P}+\mathrm{s}} T_{1}}{\partial z^{2 \mathrm{P}} \partial t^{\mathrm{s}}}\right)_{z_{\mathrm{i}}}=\frac{1}{V^{2 \mathrm{P}}} \frac{d^{2 \mathrm{P}+\mathrm{s}} T_{1}\left(z_{\mathrm{i}}\right)}{d \tau^{2 \mathrm{P}+\mathrm{s}}}
$$

これを(32)式で示した無次元座標系で表わすと，

$$
\left(\frac{\partial^{2 \mathrm{P}+} \theta_{1}}{\partial z^{2 \mathrm{P} \partial t \mathrm{~s}}}\right)=\frac{1}{V^{2 \mathrm{P}}} \frac{d^{2 \mathrm{P}+\mathrm{s}} \theta_{1 \mathrm{i}}}{d t^{2 \mathrm{P}+\mathrm{s}}}
$$

となる。ここに, $V$ は $V r_{1} / a_{2}$ であり, 無次元座標系 で表わした等温線移動速度である。

(83) 式を(76)，(77)式に代入すると, 次のような実 験データに対応する計算式を得る。

$$
\begin{aligned}
& \theta_{2}\left(z_{\mathrm{i}}, t\right)=\sum_{\mathrm{p}=0}^{\infty} \sum_{\mathrm{i}=0}^{\infty} \frac{M_{\mathrm{Ps}}}{V^{2 \mathrm{P}}} \frac{d^{2 \mathrm{P}+\mathrm{s}} \theta_{1 \mathrm{i}}}{d t^{2 \mathrm{P}+\mathrm{s}}} \\
& Q_{2}\left(z_{\mathrm{j}}, t\right)=\sum_{\mathrm{p}=0}^{\infty} \sum_{i=0}^{\infty} \frac{N_{\mathrm{Ps}}}{V^{2 \mathrm{P}}} \frac{d^{2 \mathrm{P}+\mathrm{s}} \theta_{1 \mathrm{i}}}{d t^{2 \mathrm{P}+\mathrm{s}}}
\end{aligned}
$$

もしも， $\theta_{1 \mathrm{i}}(1)$ の微分計算がわずらわしいのであれ ば, 次のような合理的な公式を使えばよい。

$$
\begin{aligned}
& \theta_{2}\left(z_{\mathrm{i}}, t\right)=\sum_{\mathrm{n}=0}^{\infty} K_{\mathrm{n}} \frac{d^{\mathrm{n}} \theta_{1 \mathrm{i}}}{d t^{\mathrm{n}}} \\
& Q_{2}\left(z_{\mathrm{i}}, t\right)=\sum_{\mathrm{n}=0}^{\infty} P_{\mathrm{n}} \frac{d^{\mathrm{n}} \theta_{1 \mathrm{i}}}{d t^{\mathrm{n}}}
\end{aligned}
$$

ここで $K_{\mathrm{n}}, P_{\mathrm{n}}$ は時間に依存しない係数である。

$$
\begin{aligned}
& K_{\mathrm{n}}=\sum_{\mathrm{i}=0}^{\operatorname{ent}(\mathrm{n} / 2)} \frac{M_{\mathrm{i}, \mathrm{n}-2 \mathrm{i}}}{V^{\ddot{z}_{\mathrm{i}}}} \\
& P_{\mathrm{n}}=\sum_{\mathrm{i}=0}^{\mathrm{ent}(\mathrm{n} / 2)} \frac{N_{\mathrm{i}, \mathrm{n}-2 \mathrm{i}}}{V^{2 \mathrm{i}}}
\end{aligned}
$$

(86)，(87)式，あるいは同じような無限級数で表わ
される式を使ら上で，その項数を簡単化のため制限し てしまうことは計算結果に常に良くないということを 頭に入れておく必要がある。

今, $t=t_{\mathrm{j}}$ が考察点であるとすれば, $t \leqslant t_{\mathrm{j}}, t \gg t_{\mathrm{j}}$ の 領域については 実験関数 $\theta_{1 \mathrm{i}}(t)$ を近似する必要があ る。なぜならば，近似関数を導入することによって， 二次微分あるいはより高次導関数を求める上で正確に なるからである。

さらに言えば, $\theta_{1 \mathrm{i}}(t)$ は近似パラメータで表わせる ベきであり，(86)，(87)式の計算には通常の級数理論 で求められるべきである。

\section{謝 辞}

著者はこの論文内容を支持して下さった安河内教授 に感謝すると共に, 論文出版の翻訳において援助して 下さった小笠原助教授及び Komakovski 氏に深く感 謝する次第です。

\section{参 考 文 献}

1) A. G. Tyomkin : "Study of conductivity by inverse methods," Moscow, "Energiya” (1973)

2) V. K. Koshkin and E. K. Kalinin : "Unsteady heat transfer", Moscow, "Machinostroenie" (1973)

3) O. Buggraff : "Exact solution of inverse problem in heat transfer theory", "Teploperedacha" (1964) t. C. -86 , No.3 (Trans. ASME)

4) E. Sperrow and others: "Inverse problem of unsteady heat conductivity", "Prikeudnaya mekhanika" (1964) t. E-86, No. 3 (Trans. ASME)

5) O.M. Alifanov : "Obtaining boundary heat conditions by solution of inverse problem of heat conductivity" Moscow, "Machinostroenie" (1965) 